\title{
Etude assistée par ordinateur du comportement dynamique d'un bras manipulateur
}

\section{Computer-assisted study of the dynamic behavior of a}

manipulator arm

\section{Trésor Kanyiki ${ }^{1}$, François Ntambwe ${ }^{2}$}

${ }^{1}$ Département d'électromécanique, Faculté polytechnique, Université de Lubumbashi, République démocratique du Congo, tresorkanyiki@gmail.com

${ }^{2}$ Département d'électromécanique, Faculté polytechnique, Université de Lubumbashi, République démocratique du Congo, k_ntambwe@yahoo.fr

RÉSUMÉ. Le présent article a pour objectif d'analyser une solution numérique pour résoudre les équations différentielles qui décrivent le comportement dynamique d'un système multicorps. La mécanique souffre des coûts expérimentaux élevés, dans certaines circonstance, il arrive que l'ingénieure soit dans l'incapacité de tester un modèle, par exemple dans l'ingénierie aérospatiale, il est difficile de créer les conditions dans lesquelles le prototype doit évoluer. Les outils de simulation sont incontournables et sont devenus un système d'ingénierie concurrentielle. La dynamique des systèmes mécaniques joue un rôle fondamental dans l'établissement d'une relation entre les causes et les réactions qui en résultent. Dans cet article, nous avons présenté la méthode de Lagrange pour établir les équations différentielles qui décrivent les comportements dynamiques d'un système multicorps, en les appliquant au bras manipulateur. La résolution analytique par les méthodes classiques s'avère ardue, les méthodes numériques sont incontournables ; par ailleurs, le calcul d'une itération par les méthodes numériques peut prendre plusieurs heures manuellement, l'utilisation de logiciel de simulation est indispensable. Dans ce présent article, nous avons exploité le programme EasyDyn qui utilise la méthode numérique de Newmark.

ABSTRACT. This article analyzes a numerical solution to solve the differential equations that describe the dynamic behavior of a multibody system. Mechanics suffer from high experimental costs, in some circumstances, sometimes the engineer is unable to test a model, for example in aerospace engineering, it's hard to create the conditions under the prototype has to evolve. Simulation tools are essential and have become a competitive engineering system. The dynamic of mechanical systems play a fundamental role in establishing a relationship between the causes and the resulting reactions. In this article, we presented Lagrange's method to establish the differential equations that describe dynamic behaviors of a multibody system, to applying them to the manipulator arm. Analytical resolution by classical methods proves difficult, numerical methods are essential; furthermore, calculation of one iteration by numerical methods can take several hours manually; the use of simulation software is essential. In this article, we have exploited Easydy software who uses the numerical method Newmark.

MOTS-CLÉS. Bras manipulateur, Easydyn, Newmark, matrice de transformation homogène.

KEYWORDS. Manipulator arm, Easydy, Newmark, matrix of homogeneous transformation.

\section{Introduction}

Cet article s'inscrit dans le domaine de la conception assistée par ordinateur des systèmes mécaniques. Il traite de la simulation du comportement dynamique d'un système multicorps. La dynamique des systèmes multicorps est l'étude par l'intermédiaire des outils informatiques du modèle mathématique décrivant le comportement dynamique du système 
mécanique [KOU 04]. La simulation sur ordinateur du comportement dynamique d'un système mécanique est un moyen efficace pour résoudre les équations différentielles qui décrivent le comportement dynamique du système mécanique et d'en effectuer des animations.

Grâce au développement de l'informatique dans le domaine du calcul de l'informatique, la dynamique des systèmes multicorps a fait des bonds prodigieux ; cela a permis de simuler les mécanismes jugés compliqués [DIO 12]. Par exemple, il y a dix ans, il était impensable de simuler en temps réel un modèle complet de voiture automobile ayant tous les éléments de suspension, les interactions pneu/sol, ... aujourd'hui un ordinateur est capable de le faire. Citons quelques logiciels de simulation: SIMPACK (INTEC GmbH en Allemagne) ou LMS/DADS (Leuven Measurement System), ADAMs (développé par MSI Software). Certains logiciels sont développés pour des applications particulières, comme MADYMO pour la simulation de crash ou VAMPIRE pour le ferroviaire [VER 07] ; ces logiciels sont payants, d'autres sont gratuits par exemple: le programme EasyDyn. Le programme EasyDyn est développé au service de mécanique rationnelle de la faculté polytechnique de Mons.

Le programme EasyDyn effectue la simulation du comportement dynamique d'un système multicorps, et est utilisé dans cet article pour simuler le comportement dynamique du bras manipulateur. Sous EasyDyn, la simulation du comportement dynamique des systèmes mécaniques se déroule en plusieurs étapes telle que :

1. L'établissement des équations de mouvement qui gouverne le système à l'aide de l'utilitaire $\mathrm{CAGeM}$ sous $\mathrm{MuPAD}$ ) à partir du calcul des paramètres cinématiques.

2. L'analyse numérique et résolution de ces équations (librairies EasyDyn en $\mathrm{C}++$ ).

3. Les conditionnements des résultats (Gnuplot, EasyAnim).

\section{Dynamique des systèmes multicorps}

Pour établir les équations qui décrivent le comportement dynamique des mécanismes, on fait appel à des opérations sur les vecteurs, les matrices, à des différentiations ... les paramètres de configurations qui sont les inconnues de ces équations, y interviennent de manière symbolique [GER 91]. Pour aboutir aux équations différentielles de la dynamique du système multicorps, on exprime la position et l'orientation de n'importe quel point du système multicorps en fonction des paramètres de configuration. Ces paramètres sont les inconnues lors de la simulation du comportement dynamique. Les différentes approches topologiques se différent à partir du choix qu'il faut effectuer sur les paramètres de configuration. Il s'agit: l'approche en coordonnées relatives, l'approche en coordonnées cartésiennes, l'approche en coordonnées naturelles, l'approche en coordonnées généralisées et plus récemment a été proposée l'approche aux éléments finis.

\subsection{Approche en coordonnées généralisées}

Chaque approche est arrivée à une maturité commerciale. Ainsi, MESA VERDE et SIMPACK utilisent les coordonnées relatives, ADAMS et DADS les coordonnées 
cartésiennes, COMPAMN est basé sur les coordonnées naturelles, MECANO sur les éléments finis et EASYDYN sur les coordonnées généralisées [DEH 95].

En coordonnées généralisées, la position de chaque éléments du système multicorps est décrite par un nombre des paramètres de configuration égal au nombre de degrés de liberté. Aucune équation de contrainte n'apparait explicitement dans l'établissement des équations différentielles du mouvement. Le mouvement est décrit par un système d'équations différentielles ordinaires [DEH 95].

La simulation d'un système mécanique sous EasyDyn consiste à lui fournir son modèle cinématique. Ce dernier s'établit à l'aide de la matrice de transformation homogène du centre de gravité du solide par rapport au système d'axe galiléen ou fixe [VER 09].

\section{Modélisation mathématique d'un système multicorps}

Les bras manipulateurs sont des robots programmables généralement ayant des fonctionnalités similaires à celles d'un bras humain [HER 07]. Les solides du bras manipulateur sont liés par des liaisons qui autorisent les mouvements de rotation ou linéaires. Ils sont largement utilisés dans l'industrie automobile pour assembler les pièces, on le retrouve aussi sur les ports comme aide au chargement des bateaux. Il est beaucoup utilisé dans l'industrie moderne. Le bras manipulateur de la figure 1 est utilisé comme assistant dans les travaux de soudage [NTA 07]. Il est utilisé dans cet article pour effectuer l'étude dynamique assistée par ordinateur.
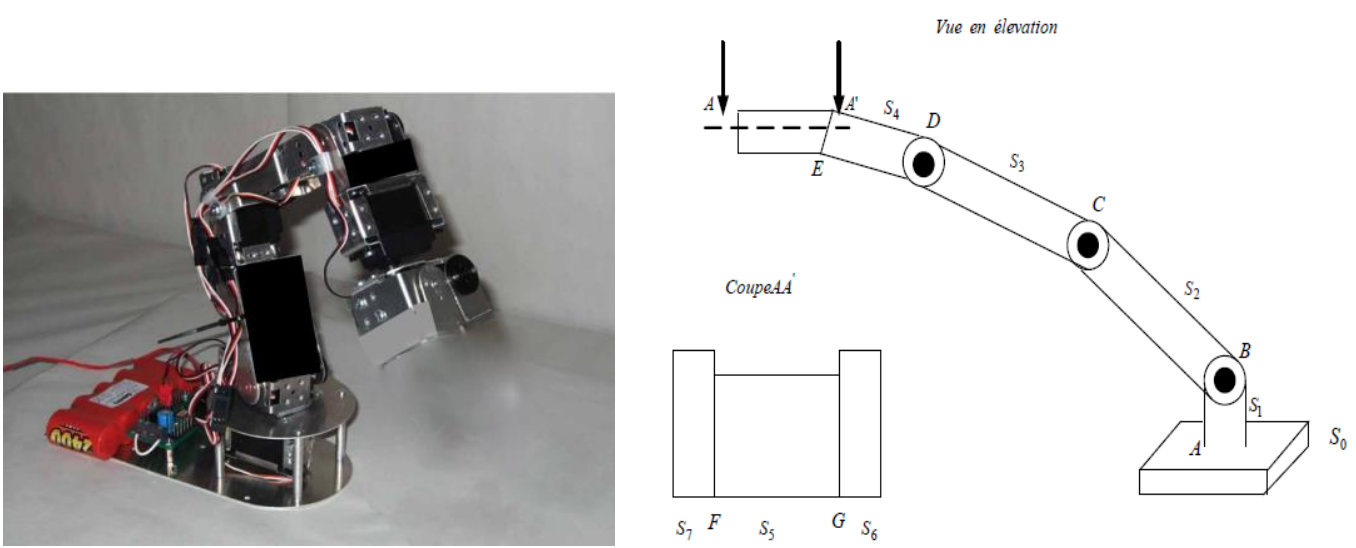

Figure 1. Bras manipulateur de type welder et sa représentation schématique

Sur la figure 1 ci-dessus, le solide $S_{1}$ représente un moteur électrique ; $S_{2}$, constitué de deux solides, $S_{2}{ }_{2}$ en aluminium et le moteur électrique $S_{2 m} ; S_{3}$ présente les mêmes caractéristiques géométriques et mécaniques que le solide $S_{2} ; S_{4}$, constitué également de deux solides $\mathrm{S}_{4}$ en aluminium et $\mathrm{S}_{4 \mathrm{~m}}$ un moteur électrique ; $\mathrm{S}_{5}$, constitué de deux moteurs électriques linéaires et enfin les solides $S_{6}$ et $S_{7}$ qui constituent l'élément terminal qui permet de tenir la porte baguette. 


\subsection{Description du mouvement de chaque solide du bras manipulateur}

La figure 2 donne une description du mouvement de chaque solide et le système d'axes orthonormés est appliqué au centre de gravité de chaque solide.

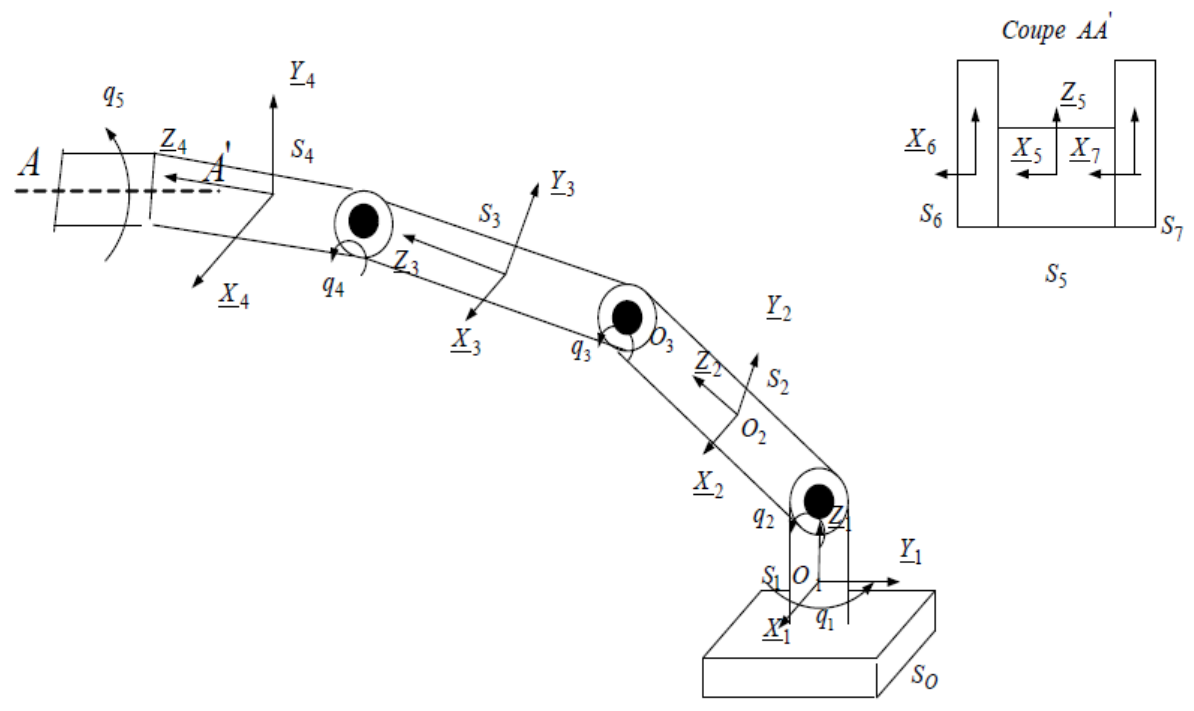

Figure 2. Description du bras manipulateur

Le modèle cinématique du bras manipulateur se présente de la manière suivante (Fig.2.6) :

- Le solide $S_{1}$ est relié au solide $S_{O}$ par une liaison rotoïde de vecteur unitaire $\underline{Z}_{1} ; q_{1}$ est le déplacement angulaire du solide $S_{1}$ autour du vecteur unitaire $\underline{Z}_{1}$. $\underline{X}_{1^{\prime}}, \underline{Y}_{1^{\prime}}, \underline{Z}_{1^{\prime}}$ sont les vecteurs unitaires orthonormés liés à la liaison rotoïde des solides $\mathrm{S}_{\mathrm{O}}$ et $\mathrm{S}_{1}$.

- Le solide $S_{2}$ est relié au solide $S_{1}$ par une liaison rotoïde de vecteur unitaire $\underline{X}_{2^{\prime}} ; q_{2}$ est le déplacement angulaire du solide $S_{2}$ autour du vecteur unitaire $\underline{X}_{2^{\prime}} \cdot \underline{X}_{2^{\prime}}, \underline{Y}_{2^{\prime}}, \underline{Z}_{2^{\prime}}$ sont les vecteurs unitaires orthonormés liés à la liaison rotoïde des solides $S_{1}$ et $S_{2}$.

- Le solide $S_{3}$ est relié au solide $S_{2}$ par une liaison rotoïde de vecteur unitaire $\underline{X}_{3^{\prime}} ; q_{2}$ est le déplacement angulaire du solide $\mathrm{S}_{3}$ autour du vecteur unitaire $\underline{X}_{3^{\prime}} \cdot \underline{X}_{3^{\prime}}, \underline{Y}_{3^{\prime}}, \underline{Z}_{3^{\prime}}$ sont les vecteurs unitaires orthonormés liés à la liaison rotoïde des solides $S_{2}$ et $S_{3}$.

- Le solide $S_{4}$ est relié au solide $S_{3}$ par une liaison rotoïde de vecteur unitaire $\underline{X}_{4}$; le déplacement angulaire du solide $\mathrm{S}_{4}$ autour du vecteur unitaire $\underline{X}_{4}$ est $q_{4}$. $\underline{X}_{4}, \underline{Y}_{4}, \underline{Z}_{4}$ sont les vecteurs unitaires orthonormés liés à la liaison rotoïde des solides $\mathrm{S}_{3}$ et $\mathrm{S}_{4}$.

- Le solide $S_{5}$ est relié au solide $S_{4}$ par une liaison rotoïde de vecteur unitaire $\underline{Z}_{5^{\prime}}$; le déplacement angulaire $q_{5}$ du solide $S_{5}$ autour du vecteur unitaire $\underline{Z}_{5^{\prime}} ; \underline{X}_{5^{\prime}}, \underline{Y}_{5^{\prime}}, \underline{Z}_{5^{\prime}}$ sont les vecteurs unitaires orthonormés liés à la liaison rotoïde des solides $S_{4}$ et $S_{5}$. 
- Le solide $S_{6}$ est relié au solide $S_{5}$ par une liaison prismatique de vecteur unitaire $\underline{X}_{6}$; le déplacement linéaire $q_{6}$ du solide $\mathrm{S}_{6}$ suivant le vecteur unitaire $\underline{X}_{6^{\prime}} ; \underline{X}_{6^{\prime}}, \underline{Y}_{6}, \underline{Z}_{6^{\prime}}$ sont les vecteurs unitaires orthonormés liés à la liaison prismatique des solides $\mathrm{S}_{5}$ et $\mathrm{S}_{6}$.

- Le solide $S_{7}$ est relié au solide $S_{5}$ par une liaison prismatique de vecteur unitaire $\underline{X}_{7^{\prime}}$; le déplacement linéaire $q_{7}$ du solide $\mathrm{S}_{7}$ suivant le vecteur unitaire $\underline{X}_{7^{\prime}} ; \underline{X}_{7^{\prime}}, \underline{Y}_{7^{\prime}}, \underline{Z}_{7^{\prime}}$ sont les vecteurs unitaires orthonormés liés à la liaison prismatique des solides $\mathrm{S}_{5}$ et $\mathrm{S}_{7}$.

\subsubsection{Paramètres de configuration du bras manipulateur}

Le bras manipulateur est constitué des sept paramètres de configuration, c'est un système holonome scléronome. Les différentes liaisons autorisent les mouvements suivants: $q_{1} \in\left[0,360^{\circ}\right] \quad, \quad q_{2} \in\left[0,180^{\circ}\right] \quad, \quad q_{3} \in\left[0,180^{\circ}\right], \quad q_{4} \in\left[0,180^{\circ}\right], q_{5} \in\left[0,360^{\circ}\right] \quad, \quad q_{6} \in[0.2,0.5]$ et $q_{7} \in[-0.2,-0.5]$. Les déplacements des solides sur le bras manipulateur sont engendrés par les actionnaires électriques, nous avons des moteurs à mouvement circulaire et les moteurs linéaires. Pour effectuer l'étude dynamique, les moteurs sont considérés homogènes et isotropes. Les caractéristiques géométriques sont représentées dans le tableau 1 [NTA 17]:

\begin{tabular}{|c|l|l|l|l|}
\hline & Largeur (b) & Longueur (a) & Hauteur (c) & masse \\
\hline Moteur électrique & $0.15 \mathrm{~m}$ & $0.35 \mathrm{~m}$ & $0.30 \mathrm{~m}$ & $58.32 \mathrm{Kg}$ \\
\hline Moteur linéaire & $0.11 \mathrm{~m}$ & $0.20 \mathrm{~m}$ & $0.40 \mathrm{~m}$ & $31.185 \mathrm{Kg}$ \\
\hline
\end{tabular}

Tableau 1. Caractéristiques géométriques des moteurs du bras manipulateur

Les efforts générés par les actionnaires pour déplacer les solides du bras manipulateur sont consignés dans le tableau 2 [NTA 17].

\begin{tabular}{|l|l|}
\hline Moteur électrique 1 & Efforts développés \\
\hline Moteur électrique 2, 3 et 4 & $0-5000 \mathrm{Ncm}$ \\
\hline Moteur électrique 5 & $0-35000 \mathrm{Ncm}$ \\
\hline Moteur électrique 6 et 7 & $0-297 \mathrm{Ncm}$ \\
\hline
\end{tabular}

Tableau 2. Efforts générés par les actionnaires électriques

\subsubsection{Caractéristiques géométriques des solides du bras manipulateur}

Les solides du bras manipulateur sont entrainés par les actionnaires électriques, ces solides sont hétérogènes constitués d'une partie en aluminium et l'autre partie est l'actionnaire. Sur le tableau 3, nous avons les caractéristiques géométriques des solides [NTA 17]. 


\begin{tabular}{|c|c|c|c|c|}
\hline Solides & Largeur (b) & Longueur (a) & Hauteur (c) & masse \\
\hline $\mathbf{S}_{\mathbf{1}}$ & $0.15 \mathrm{~m}$ & $0.35 \mathrm{~m}$ & $0.25 \mathrm{~m}$ & $46.23 \mathrm{Kg}$ \\
\hline $\mathbf{S}_{\mathbf{2}}$ & $0.15 \mathrm{~m}$ & $0.35 \mathrm{~m}$ & $0.80 \mathrm{~m}$ & $129.195 \mathrm{Kg}$ \\
\hline $\mathbf{S}_{\mathbf{3}}$ & $0.15 \mathrm{~m}$ & $0.35 \mathrm{~m}$ & $0.80 \mathrm{~m}$ & $129.195 \mathrm{Kg}$ \\
\hline $\mathbf{S}_{4}$ & $0.15 \mathrm{~m}$ & $0.35 \mathrm{~m}$ & $0.50 \mathrm{~m}$ & $66.67 \mathrm{Kg}$ \\
\hline $\mathbf{S}_{\mathbf{5}}$ & $0.22 \mathrm{~m}$ & $0.40 \mathrm{~m}$ & $0.40 \mathrm{~m}$ & $62.37 \mathrm{Kg}$ \\
\hline $\mathbf{S}_{\mathbf{6}}$ et $\mathbf{S}_{7}$ & $0.22 \mathrm{~m}$ & $0.10 \mathrm{~m}$ & $0.50 \mathrm{~m}$ & $29.7 \mathrm{Kg}$ \\
\hline
\end{tabular}

Tableau 3. Caractéristiques géométriques des solides

\subsection{Equations différentielles du bras manipulateur}

Pour écrire les équations différentielles qui décrivent le comportement dynamique du bras manipulateur, nous devrons exprimer la position et l'orientation du centre de gravité de chaque solide du bras manipulateur en fonction des paramètres de configuration, par rapport au système d'axes fixes. Le bras manipulateur a sept degrés de liberté et sa structure cinématique est une boucle ouverte.

\subsubsection{Centre de gravité d'un solide}

Le point d'application de la résultante des forces gravitationnelles est appelé centre de gravité d'un corps ; cette notion est équivalente au concept mathématique de barycentre. Les solides $S_{2}, S_{3}$ et $S_{4}$ sont hétérogènes et constitués de deux solides, la formule (1) est utilisée pour déterminer son centre de gravité [CON 09].

$$
\left\|\underline{O_{i} G_{j}}\right\|=\frac{m_{i} \times\left\|\underline{O_{i} G_{i}}\right\|+m_{2} \times\left\|\underline{O_{i} G_{j}}\right\|}{m_{i}+m_{j}}
$$

Le tableau 4, présente les positions de centre de gravité de chaque solide; le centre de gravité de chaque solide se trouve sur son axe de symétrie et est mesuré par rapport au centre du repère lié à sa base.

\begin{tabular}{|c|c|c|c|c|c|c|c|}
\hline & $\mathrm{S}_{1}$ & $\mathrm{~S}_{2}$ & $\mathrm{~S}_{3}$ & $\mathrm{~S}_{4}$ & $\mathrm{~S}_{5}$ & $\mathrm{~S}_{6}$ & $\mathrm{~S}_{7}$ \\
\hline Hauteur (c) & $0.15 \mathrm{~m}$ & $0.80 \mathrm{~m}$ & $0.80 \mathrm{~m}$ & $0.50 \mathrm{~m}$ & $0.40 \mathrm{~m}$ & $0.50 \mathrm{~m}$ & $0.50 \mathrm{~m}$ \\
\hline $\begin{array}{c}\text { Centre de } \\
\text { gravité }\end{array}$ & $0.125 \mathrm{~m}$ & $0.43 \mathrm{~m}$ & $0.43 \mathrm{~m}$ & $0.3 \mathrm{~m}$ & $0.20 \mathrm{~m}$ & $0.25 \mathrm{~m}$ & $0.25 \mathrm{~m}$ \\
\hline
\end{tabular}

Tableau 4. Centre de gravité des solides du bras manipulateur 


\subsubsection{Propriétés d'inertie d'un solide}

Le principe d'inertie dit qu'un corps ne peut modifier par lui-même son mouvement. Dans ce travail, les mouvements des solides sont modifiés par les actionnaires électriques. Raison pour laquelle nous étudions les propriétés d'inertie des solides du bras manipulateur.

\subsubsection{Matrice d'inertie en un point}

Le tenseur d'inertie en un point $\mathrm{O}$ définit la relation qui existe entre le vecteur rotation $\underline{\omega}_{j / i}$ et le moment cinétique $\underline{L}_{j / i}$. Il est démontré en mécanique rationnelle que c'est une relation tensorielle de la forme [CON 09] :

$$
\underline{L}_{j / i}=\left[\Phi_{O}\right]_{i} \underline{\omega}_{j / i}
$$

Dans cette relation, $\left[\Phi_{o}\right]_{i}$ est le tenseur d'inertie du point $\mathrm{O}$ dans le système d'axes $i$ et elle peut se mettre sous la forme suivante [CON 09] :

$$
\left[\Phi_{O}\right]_{i}=\left(\begin{array}{ccc}
J_{O x x} & -J_{O x y} & -J_{O x z} \\
-J_{O x y} & J_{O y y} & -J_{O y z} \\
-J_{O x z} & -J_{O y z} & J_{O z z}
\end{array}\right)
$$

Dans la relation (3), $J_{O i i}$ est le moment d'inertie mécanique par rapport à l'axe $O i$ et $J_{O_{i j}}$ est le produit d'inertie mécanique par rapport aux axes Oi et Oj. Les moments et les produits d'inertie s'expriment en Kg. $\mathrm{m}^{2}$. Pour les solides homogènes, les formules d'intégration permettent d'obtenir directement les moments et produits d'inertie, et pour les solides hétérogènes on utilise le théorème des axes parallèles. Les composantes de tenseur d'inertie pour chaque solide sont résumées dans le tableau 5 :

\begin{tabular}{|l|l|l|l|l|l|l|l|l|l|l|}
\hline & $\begin{array}{l}\text { Longueur } \\
(\mathbf{a})\end{array}$ & $\begin{array}{l}\text { Largeur } \\
\text { (b) }\end{array}$ & $\begin{array}{l}\text { Hauteur } \\
\text { (c) }\end{array}$ & masse & $J_{G_{I} X X}$ & $J_{G_{Y} Y Y}$ & $J_{G_{I} Z Z}$ & $J_{G_{I} Z X}$ & $J_{G_{Z} Z Y}$ & $J_{G_{I} Y X}$ \\
\hline $\mathbf{S}_{\mathbf{1}}$ & $0.35 \mathrm{~m}$ & $0.15 \mathrm{~m}$ & $0.25 \mathrm{~m}$ & $46.23 \mathrm{~kg}$ & $0.3 \mathrm{kgm}^{2}$ & $0.7 \mathrm{kgm}^{2}$ & $0.6 \mathrm{kgm}^{2}$ & 0 & 0 & 0 \\
$\mathbf{S}_{\mathbf{2}}$ & $0.35 \mathrm{~m}$ & $0.15 \mathrm{~m}$ & $0.8 \mathrm{~m}$ & $129.2 \mathrm{~kg}$ & $7.2 \mathrm{kgm}^{2}$ & $9.3 \mathrm{kgm}^{2}$ & $1.6 \mathrm{kgm}^{2}$ & 0 & 0 & 0 \\
\hline $\mathbf{S}_{\mathbf{3}}$ & $0.35 \mathrm{~m}$ & $0.15 \mathrm{~m}$ & $0.8 \mathrm{~m}$ & $129.2 \mathrm{~kg}$ & $7.2 \mathrm{kgm}^{2}$ & $9.3 \mathrm{kgm}^{2}$ & $1.6 \mathrm{kgm}^{2}$ & 0 & 0 & 0 \\
\hline $\mathbf{S}_{\mathbf{4}}$ & $0.35 \mathrm{~m}$ & $0.15 \mathrm{~m}$ & $0.5 \mathrm{~m}$ & $66.7 \mathrm{~kg}$ & $1.6 \mathrm{kgm}^{2}$ & $2.4 \mathrm{kgm}^{2}$ & $1 \mathrm{kgm}^{2}$ & 0 & 0 & 0 \\
\hline $\mathbf{S}_{\mathbf{5}}$ & $0.30 \mathrm{~m}$ & $0.22 \mathrm{~m}$ & $0.35 \mathrm{~m}$ & $62.37 \mathrm{~kg}$ & $0.9 \mathrm{kgm}^{2}$ & $1.1 \mathrm{kgm}^{2}$ & $0.7 \mathrm{kgm}^{2}$ & 0 & 0 & 0 \\
\hline $\mathbf{S}_{\mathbf{6}}$ & $0.10 \mathrm{~m}$ & $0.22 \mathrm{~m}$ & $0.5 \mathrm{~m}$ & $29.7 \mathrm{~kg}$ & $0.7 \mathrm{kgm}^{2}$ & $0.6 \mathrm{kgm}^{2}$ & $0.11 \mathrm{kgm}^{2}$ & 0 & 0 & 0 \\
\hline $\mathbf{S}_{7}$ & $0.10 \mathrm{~m}$ & $0.22 \mathrm{~m}$ & $0.5 \mathrm{~m}$ & $29.7 \mathrm{~kg}$ & $0.7 \mathrm{kgm}^{2}$ & $0.6 \mathrm{kgm}^{2}$ & $0.11 \mathrm{kgm}^{2}$ & 0 & 0 & 0 \\
\hline
\end{tabular}

Tableau 5. Tenseurs d'inertie des solides du bras manipulateur 


\subsubsection{Matrice de transformation homogène}

L'analyse du système mécanique nécessite un outil qui permet de représenter la situation spatiale de tous les éléments du système multicorps. En pratique, on attache sur chaque solide un système d'axes ; et on exprime la situation spatiale de chaque solide par rapport au système d'axes fixes ou galiléen [VER 09].

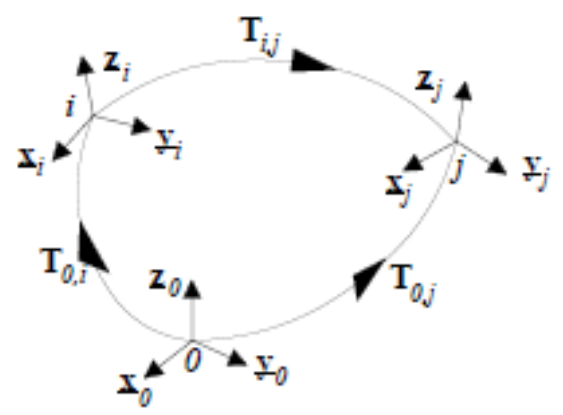

Figure 3. Matrice de transformation homogène

Le formalisme de matrice de transformation homogène permet de représenter la situation spatiale d'un solide par rapport à un autre. De ce fait, la situation relative du système d'axes $j$ par rapport au système d'axes $i$ est exprimée par la matrice de transformation homogène $T_{i j}$ de dimension $4 * 4$, qui peut être écrit comme suit [VER 09] :

$$
T_{i j}=\left(\begin{array}{cc}
R_{i, j} & \left\{\underline{r}_{j / i}\right\}_{i} \\
000 & 1
\end{array}\right)
$$

Où

- $\underline{r}_{j / i}$ est le vecteur des coordonnées du système d'axes $j$ par rapport au système d'axes $i$

- $R_{i, j}$ est le tenseur de rotation décrivant l'orientation du système d'axes $j$ par rapport au système d'axes $i$.

Les colonnes de $R_{i, j}$ correspond à exprimer les vecteurs unitaires $\underline{X}_{j}, \underline{Y}_{j}$ et $\underline{Z}_{j}$ dans le système d'axes $j$.

$$
R_{i, j}=\left(\left\{\underline{X}_{j}\right\}_{i}\left\{\underline{Y}_{j}\right\}_{i}\left\{\underline{Z}_{j}\right\}_{i}\right)
$$

Mentionnons que la matrice de transformation homogène présente l'avantage d'utiliser la propriété de la multiplication en cascade des matrices de transformation homogène.

$$
T_{i, k}=T_{i, j} \cdot T_{j, k}
$$

Cette propriété est utilisée pour obtenir la matrice de transformation homogène complexe de matrice de transformation homogène simple [GER 91]. 
Pour déterminer les matrices de transformation homogène de chaque solide par rapport au bâti fixe, nous utilisons les formules (4), (5) et (6). La multiplication en cascade des matrices de transformation homogène est réalisée lorsqu'il s'agit de trouver la matrice de transformation homogène d'un solide en passant par d'autres solides. Ainsi, on obtient :

-La matrice de transformation homogène du solide 1 par rapport au bâti

$$
T_{01}=\left(\begin{array}{cccc}
\cos q & -\sin q & 0 & 0 \\
\sin q & \cos q & 0 & 0 \\
0 & 0 & 1 & 0.125 \\
0 & 0 & 0 & 1
\end{array}\right)
$$

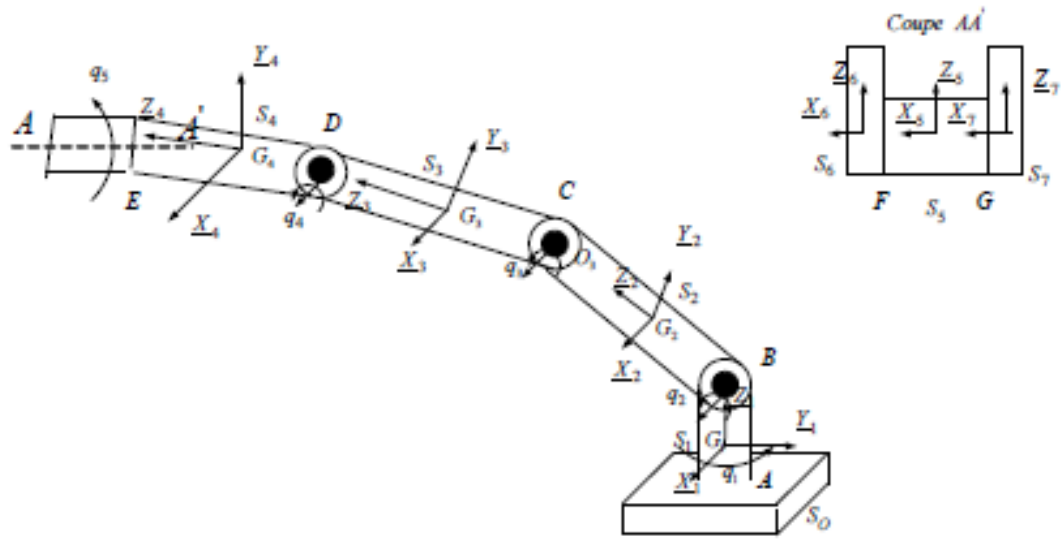

Figure 4. Schéma descriptif du bras manipulateur

-La matrice de transformation homogène du solide 2 par rapport au bâti

$$
T_{02}=\left(\begin{array}{cccc}
\cos q_{1} & -\cos q_{2} \sin q_{1} & \sin q_{1} \sin q_{2} & 0.431 \sin q_{1} \sin q_{2} \\
\sin q_{1} & \cos q_{1} \cos q_{2} & -\sin q_{2} \cos q_{1} & -0.431 \cos q_{1} \sin q_{2} \\
0 & \sin q_{2} & \cos q_{2} & 0.25+0.431 \cos q_{2} \\
0 & 0 & 0 & 1
\end{array}\right)
$$

-La matrice de transformation homogène du solide 3 par rapport au bâti

$T_{O 3}=\left(\begin{array}{cccc}\cos q_{1} & -\sin q_{1} \cos \left(q_{2}+q_{3}\right) & \sin q_{1} \sin \left(q_{2}+q_{3}\right) & \sin q_{1}\left[0.8 \sin q_{2}+0.4 \sin \left(q_{2}+q_{3}\right)\right] \\ \sin q_{1} & \cos q_{1} \cos \left(q_{2}+q_{3}\right) & -\cos q_{1} \sin \left(q_{2}+q_{3}\right) & -\cos q_{1}\left[0.8 \sin q_{2}+0.4 \sin \left(q_{2}+q_{3}\right)\right] \\ 0 & \sin \left(q_{2}+q_{3}\right) & \cos \left(q_{2}+q_{3}\right) & 0.25+0.8 \cos q_{2}+0.4 \cos \left(q_{2}+q_{3}\right) \\ 0 & 0 & 0 & 1\end{array}\right)$

-La matrice de transformation homogène du solide 4 par rapport au bâti 
$T_{O 4}=\left(\begin{array}{cccc}\cos q_{1} & -\sin q_{1} \cos \left(q_{2}+q_{3}\right) & \sin q_{1} \sin \left(q_{2}+q_{3}+q_{4}\right) & \sin q_{1}\left[0.268 \sin \left(q_{2}+q_{3}+q_{4}\right)+0.8 \sin q_{2}+0.8 \sin \left(q_{2}+q_{3}\right)\right] \\ \sin q_{1} & \cos q_{1} \cos \left(q_{2}+q_{3}\right) & -\cos q_{1} \sin \left(q_{2}+q_{3}+q_{4}\right) & -\cos q_{1}\left[0.268 \sin \left(q_{2}+q_{3}+q_{4}\right)+0.8 \sin q_{2}+0.8 \sin \left(q_{2}+q_{3}\right)\right] \\ 0 & \sin \left(q_{2}+q_{3}\right) & \cos \left(q_{2}+q_{3}+q_{4}\right) & 0.25-0.268 \sin \left(q_{2}+q_{3}+q_{4}\right)+0.8 \cos q_{2}+0.8 \cos \left(q_{2}+q_{3}\right) \\ 0 & 0 & 0 & 1\end{array}\right)$

-La matrice de transformation homogène du solide 5 par rapport au bâti

$$
\begin{array}{r}
T_{O 5}=\left(\begin{array}{cc}
\cos q_{5} \cos q_{1}-\sin q_{1} \sin q_{5} \cos \left(q_{2}+q_{3}+q_{4}\right) & -\cos q_{1} \sin q_{5}-\sin q_{1} \cos q_{5} \cos \left(q_{2}+q_{3}+q_{4}\right) \\
\sin q_{1} \cos q_{5}+\sin q_{5} \cos q_{1} \cos \left(q_{2}+q_{3}+q_{4}\right) & -\sin q_{1} \sin q_{5}+\cos q_{1} \cos q_{5} \cos \left(q_{2}+q_{3}+q_{4}\right) \\
\sin q_{5} \sin \left(q_{2}+q_{3}+q_{4}\right) & \cos q_{5} \sin \left(q_{2}+q_{3}+q_{4}\right) \\
0 & 0 \\
\sin q_{1} \sin \left(q_{2}+q_{3}\right) & \sin q_{1}\left[0.8 \sin q_{2}+0.8 \sin \left(q_{2}+q_{3}\right)+0.7 \sin \left(q_{2}+q_{3}+q_{4}\right)\right] \\
-\cos q_{1} \sin \left(q_{2}+q_{3}+q_{4}\right) & -\cos q_{1}\left[0.8 \sin q_{2}+0.8 \sin \left(q_{2}+q_{3}\right)+0.7 \sin \left(q_{2}+q_{3}+q_{4}\right)\right] \\
\cos \left(q_{2}+q_{3}+q_{4}\right) & 0.25+0.8 \cos q_{2}+0.8 \cos \left(q_{2}+q_{3}\right)-0.3 \cos \left(q_{2}+q_{3}+q_{4}\right) \\
0 & 1
\end{array}\right)
\end{array}
$$

\section{-La matrice de transformation homogène du solide 6 par rapport au bâti}

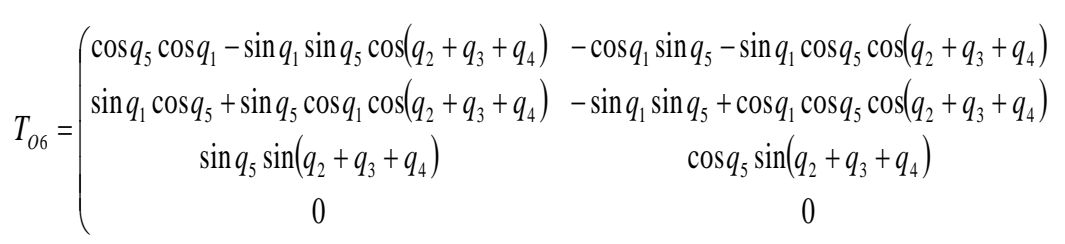

$\left.\sin q_{1} \sin \left(q_{2}+q_{3}\right) \quad-\left(0.4+q_{6}\right)\left[\cos q_{5} \cos q_{1}-\sin q_{1} \sin q_{5} \cos \left(q_{2}+q_{3}+q_{4}\right)\right]+\sin q_{1}\left[0.8 \sin q_{2}+0.8 \sin \left(q_{2}+q_{3}\right)+0.7 \sin \left(q_{2}+q_{3}+q_{4}\right)\right]\right)$ $-\cos q_{1} \sin \left(q_{2}+q_{3}+q_{4}\right)-\left(0.4+q_{6}\right)\left[\sin q_{5} \cos q_{1}-\sin q_{1} \cos q_{5} \cos \left(q_{2}+q_{3}+q_{4}\right)\right]-\cos q_{1}\left[0.8 \sin q_{2}+0.8 \sin \left(q_{2}+q_{3}\right)+0.7 \sin \left(q_{2}+q_{3}+q_{4}\right)\right]$ $\cos \left(q_{2}+q_{3}+q_{4}\right) \quad 0.25-\left(0.4+q_{6}\right) \sin q_{5} \sin \left(q_{2}+q_{3}+q_{4}\right)+0.8 \cos q_{2}+0.8 \cos \left(q_{2}+q_{3}\right)+0.2 \cos \left(q_{2}+q_{3}+q_{4}\right)$

0

\section{-La matrice de transformation homogène du solide 6 par rapport au bâti}

$T_{O 7}=\left(\begin{array}{cc}\cos q_{5} \cos q_{1}-\sin q_{1} \sin q_{5} \cos \left(q_{2}+q_{3}+q_{4}\right) & -\cos q_{1} \sin q_{5}-\sin q_{1} \cos q_{5} \cos \left(q_{2}+q_{3}+q_{4}\right) \\ \sin q_{1} \cos q_{5}+\sin q_{5} \cos q_{1} \cos \left(q_{2}+q_{3}+q_{4}\right) & -\sin q_{1} \sin q_{5}+\cos q_{1} \cos q_{5} \cos \left(q_{2}+q_{3}+q_{4}\right) \\ \sin q_{5} \sin \left(q_{2}+q_{3}+q_{4}\right) & \cos q_{5} \sin \left(q_{2}+q_{3}+q_{4}\right) \\ 0 & 0\end{array}\right.$

$\left.\sin q_{1} \sin \left(q_{2}+q_{3}\right) \quad\left(0.2+q_{6}\right)\left[\cos q_{5} \cos q_{1}-\sin q_{1} \sin q_{5} \cos \left(q_{2}+q_{3}+q_{4}\right)\right]+\sin q_{1}\left[0.8 \sin q_{2}+0.8 \sin \left(q_{2}+q_{3}\right)+0.7 \sin \left(q_{2}+q_{3}+q_{4}\right)\right]\right)$ $-\cos q_{1} \sin \left(q_{2}+q_{3}+q_{4}\right) \quad\left(0.2+q_{6}\right)\left[\sin q_{5} \cos q_{1}-\sin q_{1} \cos q_{5} \cos \left(q_{2}+q_{3}+q_{4}\right)\right]-\cos q_{1}\left[0.8 \sin q_{2}+0.8 \sin \left(q_{2}+q_{3}\right)+0.7 \sin \left(q_{2}+q_{3}+q_{4}\right)\right]$ $\cos \left(q_{2}+q_{3}+q_{4}\right) \quad 0.25+\left(0.2+q_{6}\right) \sin q_{5} \sin \left(q_{2}+q_{3}+q_{4}\right)+0.8 \cos q_{2}+0.8 \cos \left(q_{2}+q_{3}\right)+0.2 \cos \left(q_{2}+q_{3}+q_{4}\right)$

0 1 


\subsection{Equation du comportement dynamique d'un système multicorps}

La matrice de transformation homogène du solide $i$ permet de ressortir son accélération linéaire et angulaire. Les paramètres de configuration du système multicorps sont regroupés dans la matrice $\underline{q}$. Il s'agit :

$$
\begin{aligned}
& \left\{\underline{a}_{i}\right\}_{0}=\left\{J_{s_{i}}\right\}_{0} \underline{\ddot{q}}+\left\{\dot{J}_{s_{i}}\right\}_{0} \underline{\dot{q}} \\
& \{\underline{\dot{\omega}}\}_{0}=\left\{J_{\omega_{i}}\right\}_{0} \underline{q}+\left\{j_{\omega_{i}}\right\}_{0} \dot{q}
\end{aligned}
$$

Dans les expressions (14) et (15); $\left\{J_{S_{i}}\right\}_{0}$ et $\left\{J_{\omega_{i}}\right\}_{0}$ regroupent respectivement $\underline{d}_{i, j}$ et $\underline{\delta}_{i, j}$ qui désignent les contributions partielles de paramètre de configuration $q_{j}$ dans la vitesse de translation et de rotation du système d'axes $i$. Pour un système de $n_{c p}$ degré de liberté, nous avons les matrices $3 \times n_{c p}$ suivantes :

$$
\begin{aligned}
& \left\{J_{s_{i}}\right\}_{0}=\left(\begin{array}{lll}
\left\{\underline{d}_{i, 1}\right\}_{0} & \left\{\underline{d}_{i, 2}\right\}_{0} & \ldots\left\{\underline{d}_{i, n_{c p}}\right\}_{0}
\end{array}\right) \\
& \left\{J_{\omega_{i}}\right\}_{0}=\left(\begin{array}{lll}
\left\{\underline{\delta}_{i, 1}\right\}_{0} & \left\{\underline{\delta}_{i, 2}\right\}_{0} & \ldots\left\{\underline{\delta}_{i, n_{c p}}\right\}_{0}
\end{array}\right)
\end{aligned}
$$

L'équation du mouvement est obtenu en utilisant le principe de puissance virtuel (qui dérive du principe de d'Alembert) : la puissance développée par tous les efforts agissant sur le système, en incluant aussi les forces appliquées aux joints et les efforts d'inertie doit être nulle pour tous mouvement virtuel du système. Ce principe conduit à l'équation suivante en coordonnée généralisée :

$$
M(\underline{q}) \cdot \underline{\ddot{q}}+\underline{h}(\underline{q}, \underline{\dot{q}})=\underline{g}(\underline{q}, \underline{\dot{q}}, t)
$$

Avec :

M : la matrice masse de dimension $n_{c p} \times n_{c p}$, défini par :

$$
M=\sum_{i=1}^{n_{B}} m_{i}\left\{J_{s_{i}}\right\}_{0}^{T}\left\{J_{s_{i}}\right\}_{0}+\left\{J_{\omega_{i}}\right\}_{0}^{T}\left\{\Phi_{G_{\mathrm{i}}}\right\}_{0}\left\{J_{\omega_{i}}\right\}_{0}
$$

$\underline{h}$ de dimension $n_{c p} \times 1$, rassemblant les contributions de Coriolis et centrifuge, défini par :

$$
\underline{h}=\sum_{i=1}^{n_{B}}\left(m_{i}\left\{J_{S_{\mathrm{i}}}\right\}_{0}^{T}\left\{j_{s_{\mathrm{i}}}\right\}_{0}+\left\{J_{\omega_{i}}\right\}_{0}^{T}\left\{\Phi_{G_{\mathrm{i}}}\right\}_{0}\left\{j_{\omega_{i}}\right\}_{0}\right) \underline{\dot{q}}+\left\{J_{\omega_{i}}\right\}_{0}^{T}\left(\left\{\underline{\omega}_{i}\right\}_{0} \times\left\{\Phi_{G_{\mathrm{i}}}\right\}_{0}\left\{\underline{\omega}_{i}\right\}_{0}\right)
$$

$\underline{h}$ de dimension $n_{c p} \times 1$ rassemblant les contributions de force externe est donnée par :

$$
\underline{g}=\sum_{i=1}^{n_{B}}\left\{J_{s_{i}}\right\}_{0}^{T}\left\{\underline{R}_{i}\right\}_{0}+\left\{J_{\omega_{i}}\right\}_{0}^{T}\left\{\underline{M}_{i}\right\}_{0}
$$

Les matrices de transformation homogènes (7), (8), (9), (10), (11), (12) et (13) sont utilisées dans les relations (19), (20), (21) et (18) pour obtenir les équations différentielles qui décrivent le comportement dynamique du bras manipulateur. L'obtention manuelle de ces équations est fastidieuse. Ces équations différentielles non linéaires sont difficiles à résoudre par les méthodes classiques; d'où l'utilisation des méthodes numériques, d'ailleurs la résolution numérique manuelle de ces équations est ardue car on peut mettre plusieurs heures pour juste calculer une itération. L'approche numérique de Newmark est implantée 
dans le programme EasyDyn pour faciliter la résolution par ordinateur des équations de la dynamique.

\subsection{Intégration Numérique des équations du mouvement}

La résolution numérique des équations différentielles qui décrivent le comportement dynamique d'un système multicorps se fait sous EasyDyn à l'aide de la méthode numérique de Newmark. Il s'agit d'une méthode d'intégration à un pas. Partant de l'état du système à l'instant $t_{n}$ on détermine l'état du système du système à l'instant $t_{n+h}$ [VER 09].

L'équation «18» s'écrit sous la forme résiduelle suivante [BON 91]:

$$
f(\underline{q}, \underline{\dot{q}}, \underline{\ddot{q}}, t)=M(\underline{q}) \underline{\ddot{q}}+\underline{h}(\underline{q}, \underline{\dot{q}})-\underline{g}(\underline{q}, \underline{\dot{q}}, t)=\underline{0}
$$

Le comportement dynamique du système multicorps est exprimé par l'évolution temporelle des paramètres de configuration $\left(q_{i}, \dot{q}_{i}, \ddot{q}_{i}\right)$. Cela est possible par la résolution numérique des équations différentielles non linéaire du mouvement. La méthode numérique d'intégration se fait de pas en pas, le pas d'intégration est h. Pour commencer l'intégration, nous avons besoin des conditions initiales : $t^{0}, \underline{q}^{0}, \underline{\dot{q}}^{0}, \underline{q}^{0}$ et elles doivent vérifier l'équation :

$$
f\left(\underline{q}^{0}, \underline{\dot{q}}^{0}, \underline{\ddot{q}}^{0}, t^{0}\right)=\underline{0}
$$

Cependant, après chaque pas d'intégration, nous avons un système des $n_{c p}$ équations à résoudre, pour $3 \times n_{c p}$ inconnues

$$
f\left(\underline{q}^{t+h}, \underline{\dot{q}}^{t+h}, \underline{q}^{t+h}, t+h\right)=\underline{0}
$$

Par ailleurs, $2 \times n_{c p}$ équations expriment la dépendance entre chaque paramètre de configuration $q_{i}$ et ses dérivées :

$$
\begin{aligned}
& q_{i}^{t+h}=q_{i}^{t}+\int_{t}^{t+h} \dot{q}_{i} d t \\
& \dot{q}_{i}^{t+h}=\dot{q}_{i}^{t}+\int_{t}^{t+h} \ddot{q}_{i} d t
\end{aligned}
$$

Les formules d'intégrations (25) et (26) donnent une estimation de l'évolution des paramètres de configurations au cours de l'intervalle de temps. Elles prennent la forme suivante :

$$
\begin{aligned}
& \dot{q}_{i}^{t+h}=\tilde{\Lambda}\left(\dot{q}^{\leq t}, \ddot{q}^{\leq t}, \underline{q}^{t+h}\right) \\
& q_{i}^{t+h}=\Lambda\left(\underline{q}^{\leq t}, \underline{\dot{q}}^{\leq t}, \ddot{q}^{\leq t}, \ddot{q}^{t+h}\right)
\end{aligned}
$$

Le module sim d'EasyDyn réalisé l'intégration des équations u mouvement en utilisant la méthode de Newmark. Les formules de Newmark sous EasyDyn sont :

$$
\begin{gathered}
q_{i}^{t+h}=q_{i}^{t}+h \dot{q}_{i}^{t}+(0,5-\beta) h^{2} \ddot{q}_{i}^{t}+\beta h^{2} \ddot{q}_{i}^{t+h} \\
\dot{q}_{i}^{t+h}=\dot{q}_{i}^{t}+(1-\gamma) h \ddot{q}_{i}^{t}+\gamma h \ddot{q}_{i}^{t+h}
\end{gathered}
$$


Où $\beta$ et $\gamma$ sont les paramètres de la méthode de Newmark $(0,25 \leq \beta \leq 0,5$ and 0,5 $\leq \gamma \leq 1)$. Sous EasyDyn $\beta=0,25$ et $\gamma=0,5$; qui correspondent aux constantes de l'accélération entre $t$ et $t+h$ qui sont égale à la moyenne entre $\ddot{q}_{i}^{t}$ et $\ddot{q}_{i}^{t+h}$. Les formules d'intégrations sont implicites comme elle utilise l'accélération au temps $t+h$.

En remplaçant (27) et (28) dans (24), cela conduit à exprimer le système d'équations différentielles non linéaires en $\ddot{q}^{t+h}$, en vecteur de $n_{c p}$ inconnues d'accélérations et le temps $t+h$.

$$
\underline{f}\left(\underline{q}^{t+h}, \underline{\dot{q}}^{t+h}, \underline{q}^{t+h}, t+h\right)=\underline{f}\left(\Lambda, \tilde{\Lambda}, \underline{q}^{t+h}, t+h\right)=\underline{F}\left(\ddot{q}^{t+h}\right)=\underline{0}
$$

La résolution est efficace avec l'algorithme de Newton-Raphson, où le $\mathrm{n}^{\text {th }}$ estimation est calculé par :

$$
\underline{\ddot{q}}^{t+h, n}=\ddot{q}^{t+h, n-1}-J^{-1} \underline{F}\left(\underline{\ddot{q}}^{t+h, n-1}\right)
$$

Où $\mathrm{J}$ est la matrice Jacobienne (matrice itérative) de $\underline{F}$ qui respect les inconnues $\underline{\ddot{q}}^{t+h}$; les $J_{i j}$ sont définis comme suit :

$$
J_{i j}=\frac{\partial F_{i}}{\partial \ddot{q}_{j}^{t+h}}=M_{i j}+C T_{i j} \cdot \frac{\partial \tilde{\Lambda}}{\partial \ddot{q}^{t+h}}+K T_{i j} \frac{\partial \Lambda}{\partial \ddot{q}^{t+h}}
$$

\section{Simulation du comportement dynamique du bras manipulateur}

Ecrire les équations différentielles non linéaires qui décrivent le comportement dynamiques d'un système multicorps dévient fastidieux, surtout lorsque le système mécanique contient plusieurs degré de liberté. Nous le voyons avec le bras manipulateur à sept degré de liberté exploité dans cet article. Pour résoudre ces équations, nous avons utilisé le programme Easydyn. Le code se trouvant au point 4 , génère un fichier $\mathrm{C}++$; ce fichier est modifié en y ajoutant les caractéristiques géométriques du bras manipulateur, les efforts des actionnaires et les contraintes sur le paramètre de configuration. En compilant ce fichier, il donne deux fichier, le fichier .res et le fichier .vol. Le premier est utilisé par Gnuplot, pour obtenir les déplacements, les vitesses et accélérations des paramètres de configuration; le deuxième est utilisé par EasyAnim 1.3 pour visualiser les différentes animations du bras manipulateur. Cela nous donnes une idée sur la véracité des résultats. 


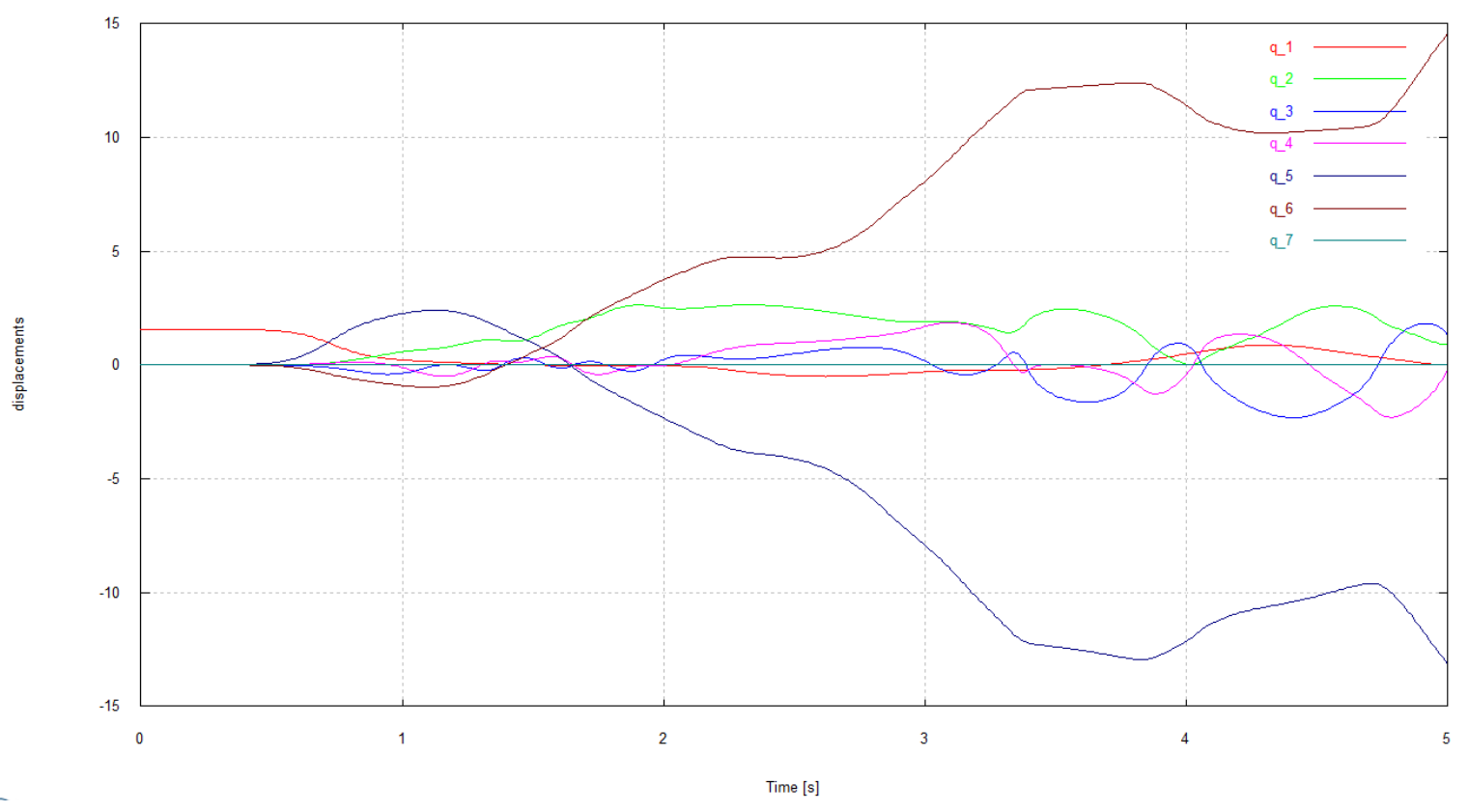

Figure 5. Evolution temporelle des paramètres de configuration

Sur les figures 5, 6 et 7 nous avons les résultats de simulation du bras manipulateur. Sur la figure 5, nous avons les amplitudes de paramètres de configuration en fonction du temps. Le temps de simulation est 5 secondes. En instant donné, nous obtenons l'amplitude de chaque paramètre de configuration, sa vitesse et son accélération grâce aux figures 5, 6 et 7 .

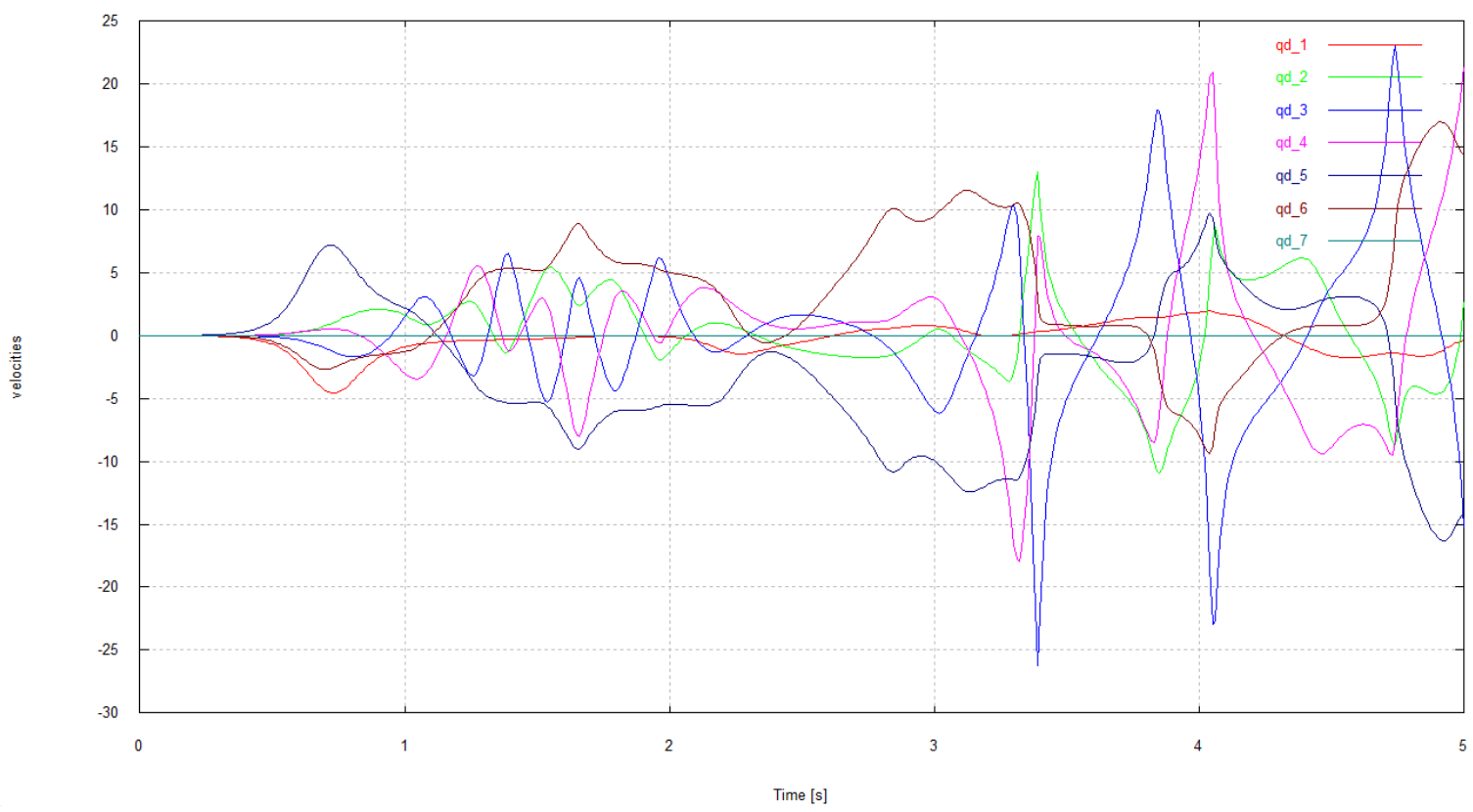

Figure 6. Evolution temporelle des dérivées premières des paramètres de configuration

Sur les figures 7 et 8 , nous avons les différentes animations du bras manipulateur pendant la simulation. Easydyn résout les équations différentielles de la dynamique, il permet de visualiser le mécanisme étudié, cette démarche permet de conditionner les résultats. 

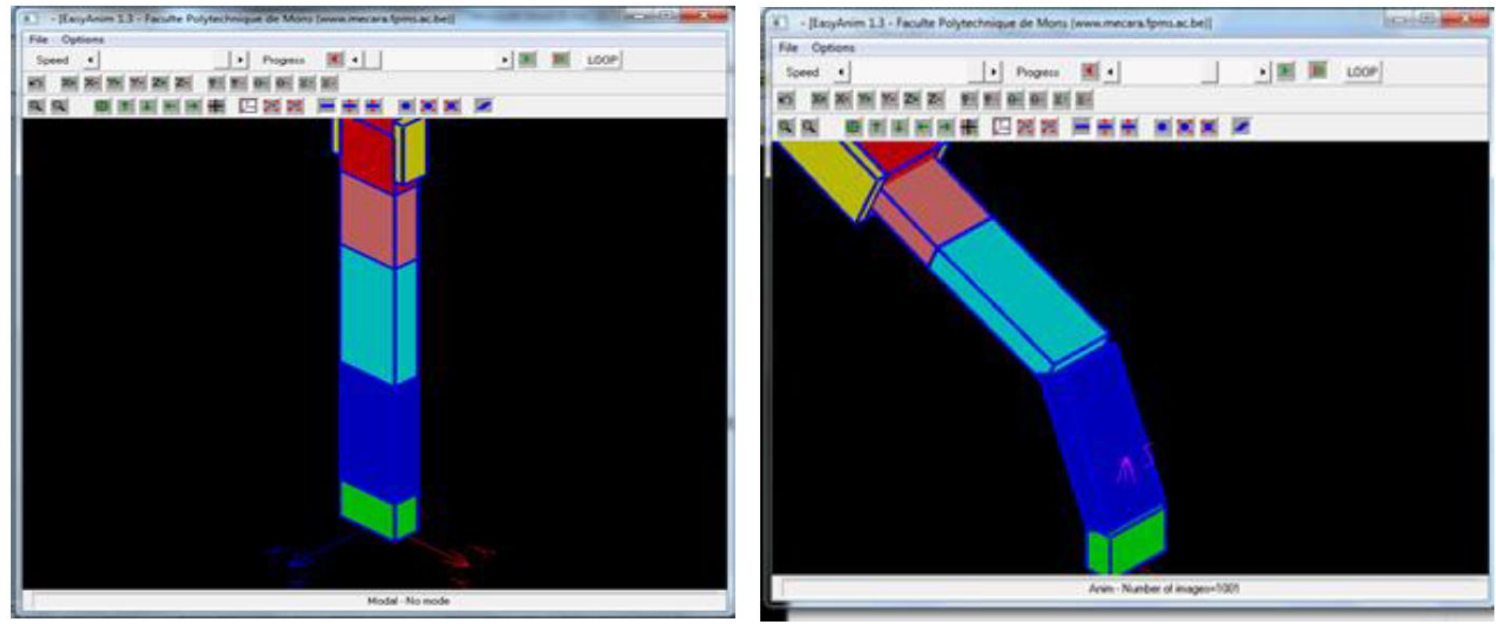

Figure 7. Animation du bras manipulateur
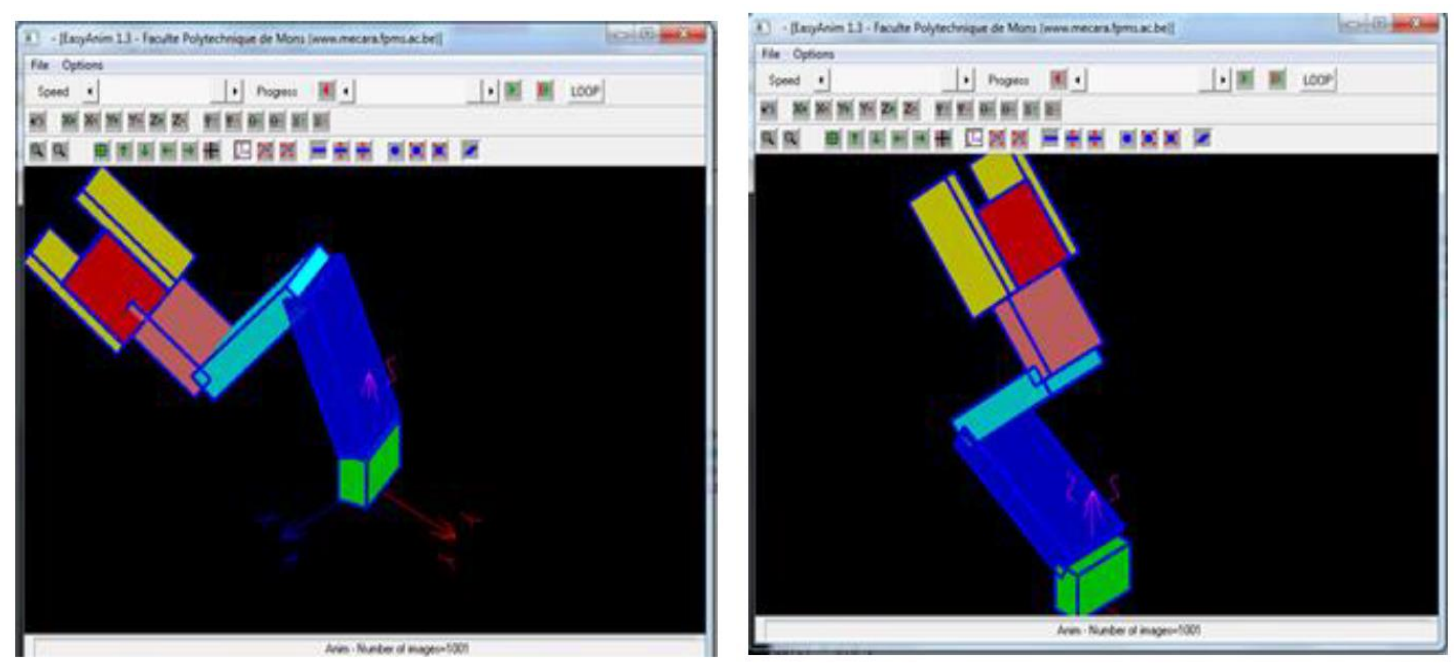

Figure 8. Animation du bras manipulateur

\section{Code Cagem}

$\mathrm{Ci}$-dessous, nous avons le code Cagem qui permet de simuler le comportement dynamique du bras manipulateur

// Titre du projet

title:="Simulation du bras manipulateur":

// Definition of nbrdof : Number of degrees of freedom

// nbrbody : Number of bodies

// nbrcont : Number of constraints (unused in this version).

nbrdof:= 7:

nbrbody:=7:

// Gravity vector 
gravity[1]:=0:

gravity[2]:=-9.81:

gravity[3]:=0:

// Eventual constants

$10:=0.25$ :

$11:=0.80$ :

$12:=0.80$ :

$13:=0.50$ :

$14:=0.40$ :

$15:=0.50$ :

$16:=0.50$ :

$\mathrm{a} 0:=0.35$ :

b0:=0.15:

b1:=0.15:

c1:=0.80:

b2: $=0.15$ :

c2:=0.80:

b3:=0.15:

c3:=0.50:

b4:=0.22:

$\mathrm{a} 4:=0.40$ :

// Inertia characteristics

mass[0]:=46.23:

$\operatorname{mass}[1]:=129.195$ :

mass[2]:=129.195:

mass[3]:=66.67:

$\operatorname{mass}[4]:=62.37$ :

$\operatorname{mass}[5]:=29.7$ :

mass[6]:=29.7:

$\operatorname{Ixx}[0]:=0.3$ :

$\operatorname{Ixx}[1]:=\left(\mathrm{b} 1^{\wedge} 2+\mathrm{c} 1^{\wedge} 2\right) / 12^{*} \operatorname{mass}[1]:$

$\operatorname{Ixx}[2]:=\left(\mathrm{b} 2^{\wedge} 2+\mathrm{c} 2^{\wedge} 2\right) / 12^{*} \operatorname{mass}[2]:$

$\operatorname{Ixx}[3]:=\left(\mathrm{b} 3^{\wedge} 2+\mathrm{c} 3^{\wedge} 2\right) / 12^{*} \operatorname{mass}[3]$ : 
$\operatorname{Ixx}[4]:=0.9:$

$\operatorname{Ixx}[5]:=0.7$ :

$\operatorname{Ixx}[6]:=0.7$ :

Iyy $[0]:=0.7$ :

Iyy[1]:=9.3:

Iyy[2]:=9.3:

Iyy[3]:=2.4:

Iyy[4]:=1.1:

Iyy[5]:=0.6:

Iyy $[6]:=0.6$ :

$\operatorname{Izz}[0]:=\left(\mathrm{b} 0^{\wedge} 2+\mathrm{a} 0^{\wedge} 2\right) / 12 * \operatorname{mass}[0]$ :

Izz[1]:=7.2:

$\operatorname{Izz}[2]:=1.6:$

$\operatorname{Izz}[3]:=1:$

Izz[4]:=(b4^2+a $\left.4^{\wedge} 2\right) / 12^{*} \operatorname{mass}[4]:$

$\operatorname{Izz}[5]:=0.11$ :

$\operatorname{Izz}[6]:=0.11$ :

// Definition of the position matrices

T0G[0] := $\operatorname{Trotz}(\mathrm{q}[0]) * \operatorname{Tdisp}(0,10 / 2,0)$ :

$\operatorname{T0G}[1]:=\operatorname{Trotz}(\mathrm{q}[0]) * \operatorname{Tdisp}(0,10,0) * \operatorname{Trotz}(\mathrm{q}[1]) * \operatorname{Tdisp}(0,11 / 2,0)$ :

$\mathrm{T} 0 \mathrm{G}[2]:=\operatorname{Trotz}(\mathrm{q}[0]) * \operatorname{Tdisp}(0,10,0) * \operatorname{Trotz}(\mathrm{q}[1]) * \operatorname{Tdisp}(0,11,0)^{*} \operatorname{Trotz}(\mathrm{q}[2]) *$ $\operatorname{Tdisp}(0,12 / 2,0)$ :

$\mathrm{T} 0 \mathrm{G}[3]:=\operatorname{Trotz}(\mathrm{q}[0]) * \operatorname{Tdisp}(0,10,0) * \operatorname{Trotz}(\mathrm{q}[1]) * \operatorname{Tdisp}(0,11,0) * \operatorname{Trotz}(\mathrm{q}[2]) *$ $\operatorname{Tdisp}(0,12,0) * \operatorname{Trotz}(\mathrm{q}[3]) * \operatorname{Tdisp}(0,13 / 2,0)$ :

$\mathrm{T} 0 \mathrm{G}[4]:=\operatorname{Trotz}(\mathrm{q}[0]) * \operatorname{Tdisp}(0,10,0) * \operatorname{Trotz}(\mathrm{q}[1]) * \operatorname{Tdisp}(0,11,0) * \operatorname{Trotz}(\mathrm{q}[2]) *$ $\operatorname{Tdisp}(0,12,0) * \operatorname{Trotz}(\mathrm{q}[3]) * \operatorname{Tdisp}(0,13,0) * \operatorname{Trotz}(\mathrm{q}[4]) * \operatorname{Tdisp}(0,14 / 2,0)$ :

$\mathrm{T} 0 \mathrm{G}[5]:=\operatorname{Trotz}(\mathrm{q}[0]) * \operatorname{Tdisp}(0,10,0) * \operatorname{Trotz}(\mathrm{q}[1]) * \operatorname{Tdisp}(0,11,0) * \operatorname{Trotz}(\mathrm{q}[2]) *$ $\operatorname{Tdisp}(0,12,0) * \operatorname{Trotz}(\mathrm{q}[3]) * \operatorname{Tdisp}(0,13,0) * \operatorname{Trotz}(\mathrm{q}[4]) * \operatorname{Tdisp}(0,14 / 2,0) * \operatorname{Trotz}(\mathrm{q}[4]) *$ $\operatorname{Tdisp}(0.2+\mathrm{q}[5], 14 / 2,0)$ :

$\operatorname{T0G}[6]:=\operatorname{Trotz}(\mathrm{q}[0]) * \operatorname{Tdisp}(0,10,0) * \operatorname{Trotz}(\mathrm{q}[1]) * \operatorname{Tdisp}(0,11,0) * \operatorname{Trotz}(\mathrm{q}[2]) *$ $\operatorname{Tdisp}(0,12,0) * \operatorname{Trotz}(\mathrm{q}[3]) * \operatorname{Tdisp}(0,13,0) * \operatorname{Trotz}(\mathrm{q}[4]) * \operatorname{Tdisp}(0,14 / 2,0) * \operatorname{Trotz}(\mathrm{q}[4]) *$ Tdisp(-0.2-q[5],14/2,0):

// Initial conditions

qi[1]:=0:

// Simulation parameters 
FinalTime: $=5$ :

StepSave: $=0.01$ :

StepMax: $=0.005$ :

SIMPLIFY:=1:

// Set FORCES to 1 in case you want to include *.AppEff.cpp into procedure

// AddAppliedEfforts() to define forces other than gravity

FORCES: $=0$ :

// Set ANIM to 1 in case you want CaGEM to generate the skeleton code

// for visualization and animation of the system

ANIM:=1:

// Set STATIC to 1 in case you want CaGEM to generate the code

// to search for static equilibrium before integration

STATIC: $=0$ :

// Set PLOT to 1 in case you want CaGEM to generate the GNUPLOT code

// to plot the evolution of position, velocity and acceeration

PLOT:=1:

// SET LATEX_FR to 1 if you want the LaTeX report in French

LATEX_FR:=1:

// SET LATEX_EN to 1 if you want the LaTeX report in English

LATEX_EN:=1:

\section{Conclusion}

Dans ce travail, nous avons présenté une approche matricielle de Lagrange pour écrire les équations différentielles qui décrivent le comportement dynamique du bras manipulateur, et la procedure de simulation.

Nous voyons les difficultés d'écrire ces équations pour un système à plusieurs dégré de liberté. Les matrices de transformations homogènes des differents solides du bras manipulateur constituent un moyen efficace pour fournir à EasyDyn le modèle cinématique $\mathrm{du}$ bras manipulateur. Après simulation, les logiciels donnent l'évolution temporelle des paramètres de configuration, de leurs vitesses et accélérations. Quant aux animations, elles permettent de conditionner le resultat et à avoir le sens physique des simulations effectuées.

\section{Bibliographie}

[BAY 05] BAYLE B., Introduction à la robotique II, Université Louis Pasteur de Strasbourg, 2005. 
[DEH 95] DEHOMBREUX P., simulation $d u$ comportement dynamique de systèmes multicorps contraints, Thèse de Doctorat, Faculté polytechnique de Mons, 1995.

[HER 07] HERRERA H, commande des bras manipulateurs et retour visuel pour des applications à la robotique de service, Thèse de Doctorat, université Toulouse III (spécialité robotique), 2007.

[CON 09] CONTI C. et BOUCHER S., cours de mécanique rationnelle II, Faculté polytechnique de Mons, 2009.

[KOP 11] KOPPER C., Principes variationnels et Mécanique analytique, école polytechnique de Montréal, 2011.

[GER 93] GERADIN M., théorie des vibrations, MASSON, 1993, page 364-405.

[GER 91] GERADIN M., Cours de dynamiques des systèmes multicorps, université de liège, 1991.

[DION 12] DION J.L, Théorie et pratique des logiciels de simulations des mécanismes "Systèmes multicorps », Institut supérieur de mécanique de paris, 2012.

[DUY 04] DUYSINX P. et GERADIN M., An introduction to robotics: mechanical aspects, university of liege, 2004.

[DIO 12] DION J.L, simulations des mécanismes ," "Systèmes multicorps" "“ : Applications en environnement ADAMS et MATLAB, Institut supérieur de mécanique de paris, 2012.

[CONTI 14] CONTI C. et BOUCHER S., cours de mécanique rationnelle I, Faculté polytechnique de Mons, 2009.

[BON 07] BONNEL H., cours d'analyse numérique, université de la Nouvelle-Calédonie, 2007.

[SHA 05] SHABANA A., Dynamics of multibody systems, university of Illinois at Chicago, 2005.

[KAB 09] KABEYA P., simulation and control of polycycle : the Anacoda project, Postgraduate advanced Degree thesis, Faculté Polytechnique de Mons, 2009.

[BOM 11] BOMBLED Q., Modeling and control of six-legged Robots: "Application to AMRU5", Thèse de Doctorat, université de Mons, 2011.

[KOU 04] KOUROSSIS G. et VERLINDEN O., cinématique et dynamique des mécanismes assistées par ordinateur : Manipulation EasyDynn, Faculté Polytechnique de Mons, 2004.

[VER 07] VERLINDEN O. et KOUROUSSIS G., EasyDyn 1.2.4 "C++ Libray for the easy simulation of dynamic problems", Faculté polytechnique de Mons, 2007.

[KAB 16] KABEYA P., Modeling, Dynamics and control of the Anaconda, Faculté polytechnique, Université de Mons, 2016.

[VER 09] VERLINDEN O., computer-aided analys of multiboby systems, Faculté Polytechnique de Mons, 2009.

[NTA 17] NTAMBUE F., cours de robotique, université de Lubumbashi, 2017. 